\title{
A Framework for Optimizing the Response Quality of Services in Mobile Cloud Computing Systems
}

\author{
Somayyeh Zahedian \\ Payam Noor University \\ Department of Computer \\ PO BOX 19395-3697, Tehran, Iran
}

\author{
Arash Ghorbannia Delavar \\ Payam Noor University \\ Department of Computer \\ PO BOX 19395-3697, Tehran, Iran
}

\author{
Yalda Aryan \\ Educational Computer Group \\ Research Center of Teachers \\ PO BOX 8134776151, Isfahan, Iran
}

\begin{abstract}
The subject of providing mobile users with an optimized service based on the Service Level Agreement (SLA) in cloud computing environment is one of the controversial issues, because there are a lot of challenging features in this environment such as the heterogeneity of cloud resources and also processing power of mobile phones.

In this article, a framework called CSRAM is proposed for optimizing the response quality of services $(\mathrm{QOS})$ in mobile cloud computing systems that tries to increase the precision and speed of the best service selection by offloading part of the computations to the cloud as well as using the context information of service provider in request adaptation process.

In the proposed framework, it was tried to design a modular system and also to consider an appropriate algorithm for using in service request adaptation process. Finally, with regarding to seven effective environmental parameters as the inputs and also, with comparison between CSRAM framework and another applied framework, more flexibility was achieved in changing the environmental parameters of the problem, Reduction in the imposed computational load on user's mobile phone and also, increase in solution precision based on the reality.
\end{abstract}

\section{General Terms}

Cloud Computing, Mobile Cloud Computing, Service Discovery.

\section{Keywords}

Mobile Cloud Computing, Service Discovery, Context Awareness, Quality of Service, Service Request Adaptation.

\section{INTRODUCTION}

The concept of mobile cloud computing was introduced shortly after launching the cloud computing platform. Since then, with the increasing use of smartphones and access to the fast and reliable wireless Internet, people are more interested in using mobile applications and also cloud computing platform to have their required services. Mobile cloud computing represents the technology of mobile services in the cloud environment.

One of the important issues in mobile cloud systems is the variable quality of service based on the change of user's context information like hardware resources, environmental variables, user preferences, etc. Receiving a service with the different quality from user expectations can lead to overuse of phone resources such as battery life and other costs, as well as his dissatisfaction [1].
One of the solutions for the above problem is using a middleware for monitoring the phone resources and context information and dynamically adapting cloud services based on that. This adaptation happens gradually so that best allocation of resources takes place every moment. Then the middleware rechecks the cloud services to make sure that the intended service is offered based on the requests in the new condition. When the running service does not have the requested quality, the service rechecking process is gone through again to provide the expected quality. Several frameworks have been offered in this regard from which one is the proposed method in [2] called VOLARE. This framework acts as an embedded middleware module in a mobile phone which starts working after the user runs an application and sends a service request to the cloud service (service discovery time). Firstly, it receives the service request and QoS and context information through the operating system and then based on this data, it goes through the processing and transmitting task, then in every moment of running the service, if the quality level of the running service is different from what actually can be offered, the process of the appropriate request adaptation and retransmission to the cloud is done. One of the advantages of this technique is the auto-detection power of the context changes of the user and offering a proper service request in the run time.

In this article, a framework called CSRAM is proposed which is an expansion to VOLARE framework for the adaptation of the mobile users' requests in cloud platform and tries to keep the real-time nature of the system and at the same time, tries to increase the speed and accuracy of response with offloading part of computations to the cloud. In addition, in the proposed framework, it was tried to use the service provider's context information as well as user's information to increase the precision of service selection process for VIP users. In VOLARE system, a mathematical model is used in the request adaptation process. Since this issue is considered as a NP-hard problem regarding to the vast number of environmental parameters, performing the request adaptation process with genetic algorithm [3] makes CSRAM framework more flexible in real-time change of problem searching area, as well as the independency of adaptation function from the type of the requested service.

In this article, the basic concepts of mobile cloud computing and previous related studies were investigated and also, the proposed framework and request adaptation algorithm were introduced. For this purpose, in section 2 the basic concepts are illustrated, in section 3 the related works are discussed, and in section 4 the proposed framework is introduced. The simulation result of CSRAM is presented in section 5, and the conclusion and suggestion for future studies in section 6 would end the article. 


\section{BASIC CONCEPTS}

Cloud computing is a paradigm for distributed computing that tries to allocate huge resources to a vast number of requests. These resources are offered on the Internet under the name of different services [4], [5] and are generally implemented in the following three layers:

- Data center layer provides the hardware infrastructure of the cloud in which some servers are connected to each other under a high speed network to provide user requested services.

- Infrastructure as a service (Iaas) is on the top of the data center layer which provides storage, hardware, servers and network components.

- Platform as a service (Paas) provides an advanced integrated environment for creating, testing and developing the applications.

- Software as a service (Saas) supports different softwares that are accessible for users via the Internet.

Cloud services are accessible through the broker that allows the client to send his request with the expected quality of service to the cloud and then, regarding to the request, it binds to the best service provider. In fact, the broker acts as an interface between the client and the cloud.

Mobile cloud computing simply refers to an infrastructure in which data storing and processing occurs out of mobile phone. It means that CPU intensive processes as well as data storing can be offloaded from the mobile handsets into the cloud; therefore, the mobile phones do not need a strong configuration (e.g. CPU speed and storage capacity). In fact, mobile cloud computing is a combination of mobile web and cloud computing that is considered as a popular tool among the users for accessing the Internet applications and services. The overall architecture of mobile cloud computing is illustrated in figure 1 [6].

Service providers in mobile cloud computing try to deliver a customized service considering the conditions and tastes of different users. They do this through monitoring the context information of users' mobile phones and also their preferences. Therefore, the QoS parameters like cost, response time, availability, etc. can distinguish them and make one service provide more outstanding than the others.

\section{RELATED WORKS}

In Recent years, the topic of web service adaptation with user's context information and providing the optimized quality of service is very important for the researchers, and different frameworks and algorithms have been proposed in this regard.

Frameworks like CARISMA [7], CHISEL [8], MUSIC [9] and PLASTIC [10] have a similar approach and try to adapt the service request based on variable context information and predefined policies. MUSIC does the adaptation process dynamically; however, it is not done based on the changes of user's context information at runtime. In fact, its adaptation criteria are based on the changes of available services at runtime.

In the proposed approaches in Q_CAD [11] and DINO [12] some frameworks are recommended for the selection of the optimum service regarding the context and QoS information. One considerable point About Q_CAD framework is that it does not support the dynamic adaptation process of service request at runtime. Further, DINO uses a broker oriented framework for adaptation process. This means that management of data processing and formation of an optimized service request is done in the broker. Adaptation mechanism in [13] is done based on a utility model definition and using the users' and the service providers' information which binds user to the best service provider. Simultaneously, it considers service provider's benefits in terms of cost, resources, etc.

[14] Approach offers a framework for a dynamic service adaptation based on the user's context information. In this approach, firstly, a service is delivered, and then at the runtime, the dynamic adaptation process based on the comparison of the user's context with the context of the primarily delivered service is done.

In the source of [15], a framework for the discovery of proactive services in pervasive environments is proposed that operates based on HAC algorithm, and its purpose is to find and rate different services with different performances proportionate the user's current context information and finally, to provide him with the best service. SMICLOUD [16] offers a framework for rating the cloud services regarding to the user expected quality of service, and it applies AHP algorithm as the weighting and service request adaptation mechanism. However, the purpose of this approach is getting a value to the different available cloud services based on the context information and preferences of the user as well.

The approach in [17] proposes a framework that provides context-aware services regarding to the detection of the change type, cause and accordingly, choose a suitable adaptor. However, the causes and the list of adaptors have been predefined which it decreases the flexibility of this approach in real world.

The approach in [18] offers a QoS-aware system for mobile cloud computing that operates based on monitoring and assessing the QoS parameters and adaptation with an appropriate QoS mode. In this approach, monitoring process is done on the phone side and adaptation process is done on the cloud side that seems logical from processing aspect.

The approach in [19] offered a framework for the quality adaptation of the service with the user status and priorities. It does this by assessing, weighting the different service parameters and user information, and finally by their comparison. It applies FCM algorithm for adaptation, but like the proposed approach in [2], it centralizes all request adaptation processes on the user mobile phone.

\subsection{VOLARE Framework}

VOLARE system performs its task in two levels of service discovery and runtime. Once the application requests a service, the service discovery phase begins. Service request module interprets this request and sends it to the adaptation module. At the same time, the context information of the user mobile phone is monitored continually, and its current context information is sent to the adaptation module. The adaptation module manages the service requests based on the current context information of the mobile phone. Then, the running phase begins. At run time, in case of a change in the value of context information, the reevaluation of the available services is done. These results in binding the application to a new service based on the new context and QoS. The level of QoS is monitored by QoS monitoring module, and if there is any difference between current QoS and expected QoS levels, 


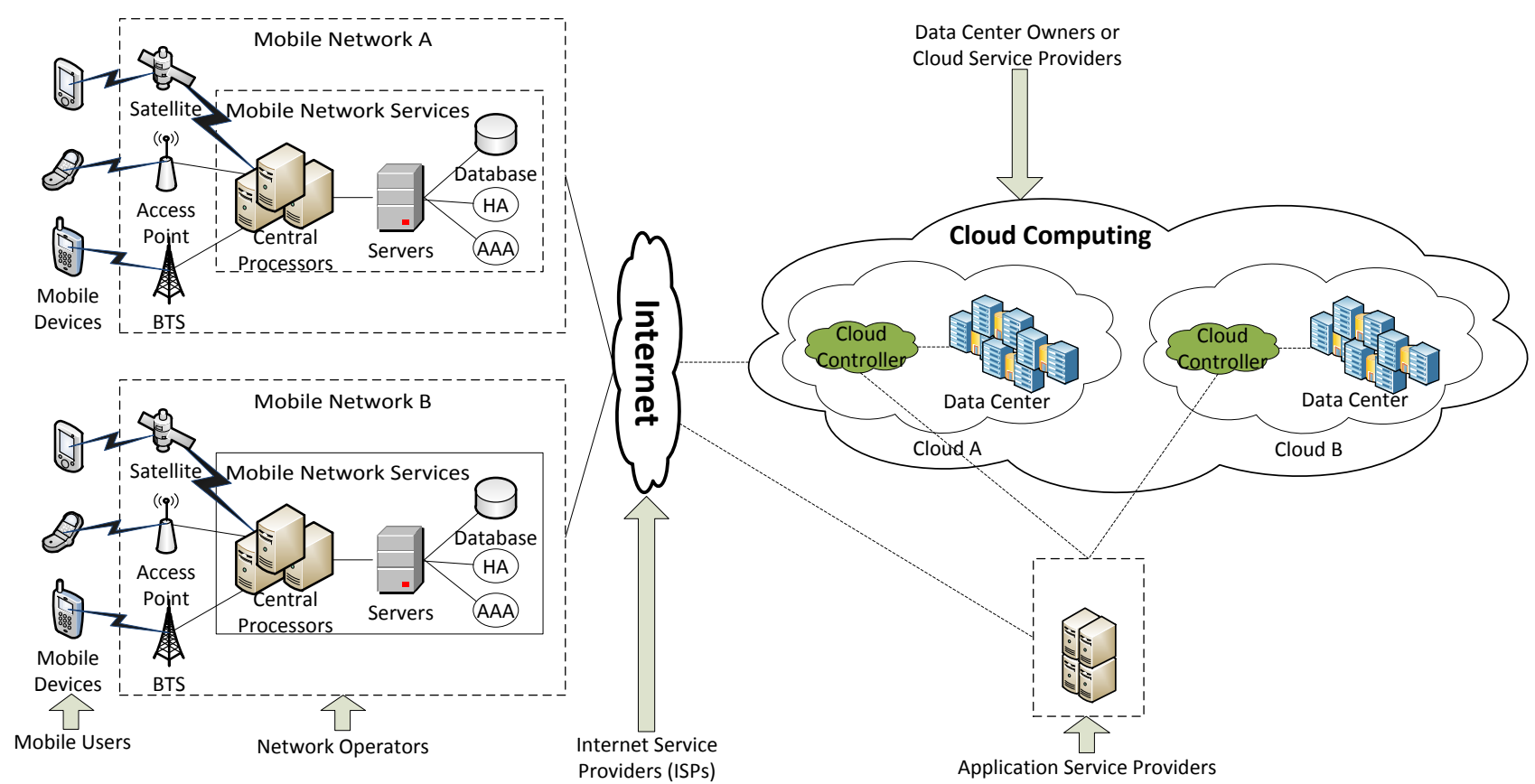

Fig 1: Mobile Cloud Computing Architecture [6]

service request module will be informed and the cycle of new service discovery begins. The processes of service discovery and also service binding is done by service binding module. This is performed through sending the adapted service request to the broker. Then, the broker selects the best service provider based on the received request.

The definition of QoS levels is done by putting an adaptation policy file in the mobile phone which is written in two policy levels i.e. global-level and service-level. Also, VOLARE framework uses mathematical model in its request adaptation process [2].

One of the advantages of the VOLARE framework is the power of monitoring and auto-detection of user context changes, and also delivering an appropriate service request dynamic adaptability of the service request at run time. However, the focus of the total process of adaptation on the mobile phone which has limited storage and processing resources, as well as, the dependency of the used mathematical model to the type of service, make it difficult to develop this framework for different applications.

\section{PROPOSED FRAMEWORK: CSRAM}

CSRAM framework for service selection based on context information and expected quality level is a middleware solution which evaluates the received service request and adapts it with regard to the context information of user and service provider, and also the QoS information. Then, it sends the adapted request to the cloud.

The purpose of the proposed framework is finding an appropriate solution for the mentioned problems. Accordingly, it was tried to keep the nature of the real-time system as well as to increase the speed and accuracy of the response by offloading the computational part of request adaptation process to the cloud. Also, it was tried to provide VIP users with a careful selection of an optimized service by using the context information of the service providers. The replacement of mathematical model used in VOLARE adaptation process with genetic algorithm which is applicable in solving NP-hard and optimization problems, has also made CSRAM framework more flexible in real-time change of problem search area, as well as in the independency of adaptation function from the type of the requested service. Figure 2 shows the proposed framework of service request management based on context and QoS information. The modular design of CSRAM makes it possible to develop it for different applications.

The proposed framework includes the following modules:

- Request management module: this module acts as an interface between the mobile phone application and the cloud and creates or reconstructs the body of the request considering the context and QoS information and finally, sends it to the broker. The fact that the request management process does not need a great processing power, and also the modular design approach of the system heightens the importance of this module being laid on the phone side.

- Context management module: this module monitors the different qualitative or quantitative context data. Then it analyses the data and convert it to the information format required by request management module.

- QoS management module: this module monitors the different QoS data. Then it analyses the data and convert it to the information format needed by request management module.

- Service management module: this module gets the body of service request and in case of a VIP user, the context information of the service provider is obtained too and then creates/reconstructs it based on the considered format. Then it sends the request to adaptation management module, and after receiving the adapted request from this module, it sends the service binding request to the best service provider. In fact, service management module acts like an 


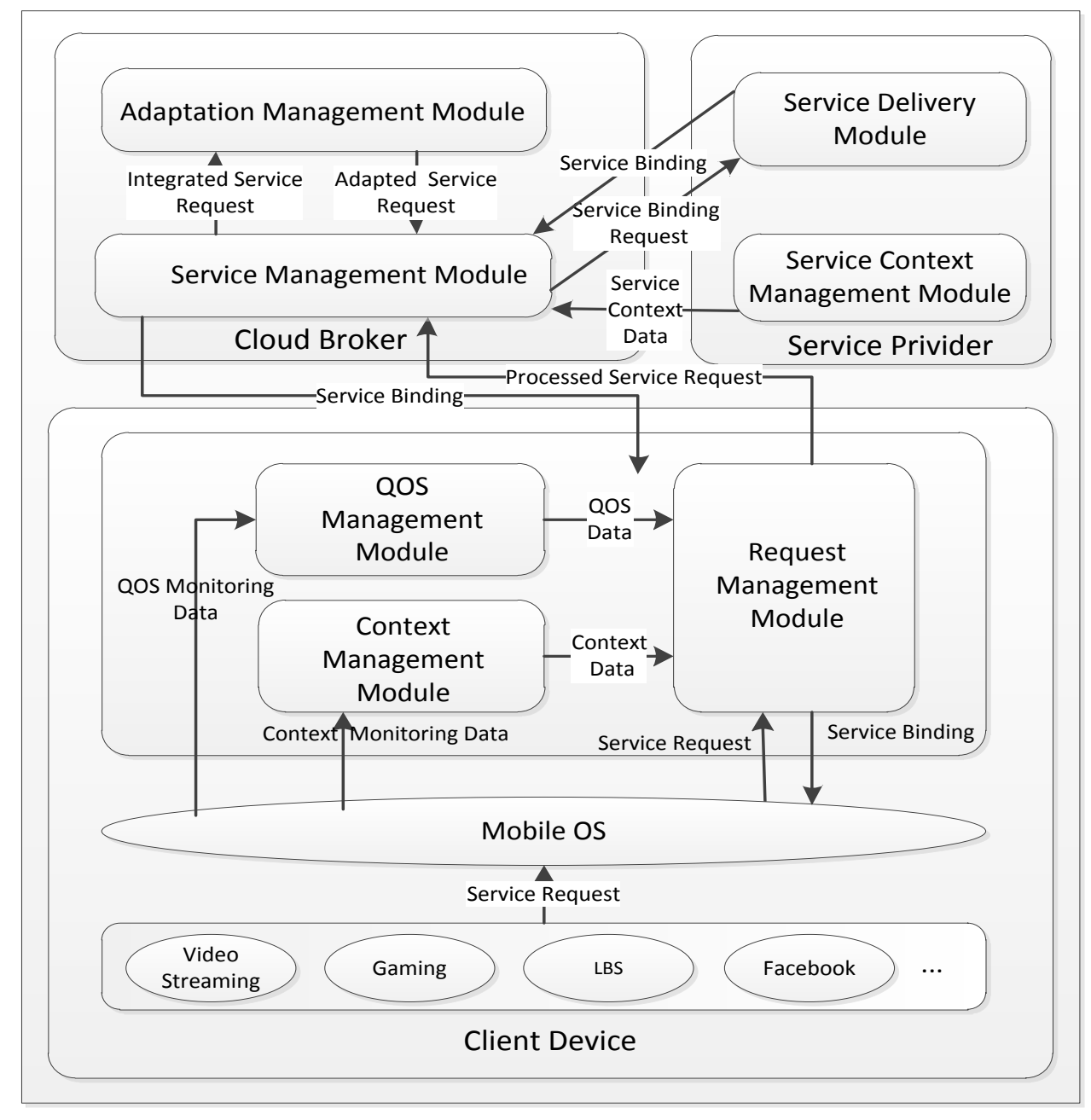

- $\quad$ Fig 2: Service request management of CSRAM

interface between the cloud and the service provider and therefore, in the modular design of the system, it is on the cloud side.

- Service context management module: this module monitors the different qualitative or quantitative context data of a service provider. Then it analyses the data and convert it to the information format needed by service management module. Using the considered data in the request adaptation process increases the degree of accuracy and real precision.

- Adaptation management module: this module adapts the service request regarding to the received data from service management. Some parameters like the limited processing power of the mobile phone and the possibility of using a wide range of adaptation algorithm in the cloud, multiplies the importance of putting this module on the cloud.

- Service delivery module: this module acts as an interface between the cloud and the service provider and provides the user with the appropriate service regarding to the received request.

\subsection{Flowchart}

As mentioned before, CSRAM framework does the processes of user request management and adaptation based on the available network quality through different stages. Figure 3 shows the flowchart of the processing and sending the adapted service request.

In the proposed framework, the service discovery phase begins right after receiving the service request from the user. In this phase, request management module receives the user context information from context management module and uses it for analyzing and processing the request received from the application. Then it transmits the processed request to service management module which is on the cloud. If the originator of the request is a VIP user, service management module asks the service providers to send the context information of their services. Then it sends the integrated service request to adaptation management module and receives the adapted request from this module. Then it does the best service selection process regarding to the provided data, and then it sends the adapted request to the considered service provider. Finally, it directs the binded service to the user.

Now, the runtime phase begins in which, the context and QoS information is monitored continually and the updated request is sent to the cloud. At the same time, the context information of the service provider is monitored and sent to the cloud. Adaptation management module collects the received data, and if there is a difference between the available quality level with expected one in the service, it retransmits the adapted request for the best service selection. 


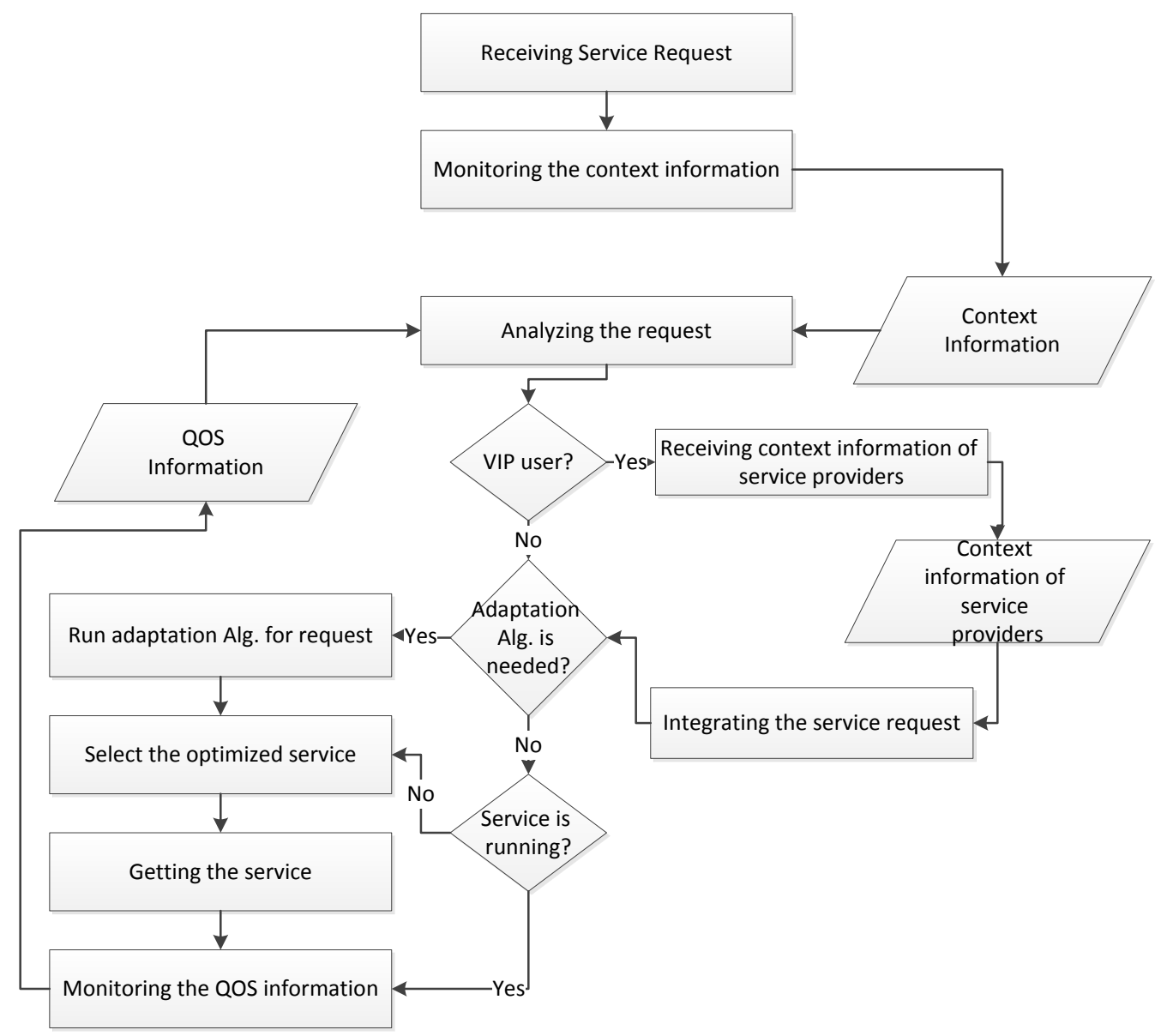

Fig 3: Flowchart of service request management process

\subsection{Adaptation Algorithm}

Since the adaptation problem is considered as an optimization issue, the related techniques like genetic algorithm can be used in this regard.

Genetic algorithm [3] is a heuristic technique for solving the NP-hard problems. Some of the advantages of genetic algorithm compared to the mathematical models are:

- The heuristic nature of the algorithm for finding the global optimum solution in the searching area

- Independence from the initial values in the process of finding the optimal solution

- Adaptation process conversion from an static algorithm to a dynamic one

- More Careful selection of the optimal service according to the user expected quality

- Using a unified template in the different applications and services

In the used genetic algorithm, each service mode is considered as a chromosome with the length of $n$. The variable $n$ indicates the number of environmental parameters. Figure 4 shows a sample of a chromosome.

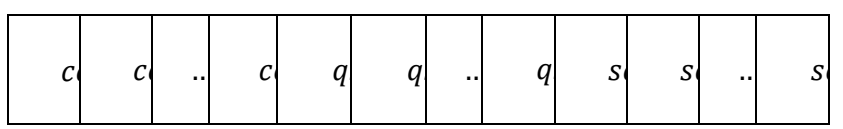

Fig 4: Sample chromosome in CSRAM adaptation Alg.
According to the above figure, the formation of the initial population is done by the chromosome which consist of effective environmental context information $\left(\mathrm{cc}_{1} \ldots \mathrm{cc}_{\mathrm{i}}\right)$, QoS parameters $\left(\mathrm{qs}_{1} \ldots \mathrm{qs}_{\mathrm{j}}\right)$ and the context information of the service providers $\left(\mathrm{sc}_{1} \ldots \mathrm{sc}_{\mathrm{k}}\right)$. Then, crossover, mutation and selection processes are repeated on the considered population until obtaining an optimum solution. The adaptation algorithm begins with the service discovery phase and continues to the end of the service run time.

The selection process of the optimum solution is done based on the fitness value of available solutions. The purpose of this article is selecting a solution with the maximum fitness value. In addition, single-point crossover operation was used on the condition that the selected gene is a QoS parameter. This condition can also fit in mutation operation.

The method of the optimal service selection is Rolette Wheel which returns the selected population in each epoch.

In the following, the pseudo code of the proposed algorithm is shown: 
Pseudo Code of Adaptation Module in CSRAM Framework Input: An integrated request and the set of Available services characteristics and

Output: An optimized service request

1. Do

2. Make initial Population

3. While the optimized solution will be obtained

4. Cycle cross over operation

5. Goal oriented mutation operation

6. $\quad$ Select the best chromosomes

7. End while

8. Dispatch the optimized solution

9. Until service is running

\section{EXPERIMENTAL RESULTS}

\subsection{Case Study}

Since there is no standard data set for evaluation in this field, VOLARE case study was used for evaluating the results of the proposed framework. In this case, the user can subscribe one of the four service modes of video streaming service (i.e. 28, 56,128 or $256 \mathrm{kbps}$ ) according to the SLA. However, because of the different network limitation, the user cannot use the preferred choice. By default, the user should pay for the agreed cost in the SLA. In fact, the purpose of CSRAM is reduction of this cost and converting it to the cost of real usage. In this section, the degree of the proposed framework effect on cost reduction will be evaluated. For simplicity, the video decoding process on the mobile phone was ignored and it was assumed that the user receives streaming packets.

\subsection{Evaluation Parameters}

The genes of each chromosome include the service and mobile phone context and also QoS parameters. At every moment, the different chromosomes of the population have the same context but different QoS values.

In order to make the selected solution closer to the reality, a set of the following parameters and criteria is used to define the fitness value of each solution in every stage. The equations (1), (2), (3) and (4) show the considered parameters and related weights, and the equation (5) shows the used fitness function.

$c c_{i} \in[$ Battery, CPU, Bandwidth], $\mathrm{i} \in[1 . .3]$

$q s_{j} \in[$ Response Bandwidth, Availability,

ResponseTime $], \mathrm{j} \in[1 . .3]$

$s c_{k} \in[$ Available $C P U], \mathrm{k}=1$

if $j=3$, then $W_{j}=\frac{1}{W_{j}}$ $\sum_{i=1}^{3} W_{i}+\sum_{j=1}^{3} W_{j}+\sum_{k=1}^{1} W_{k}=1$

Fitness $_{\text {total }}=\sum_{i=1}^{3} W_{i} c c_{i}+\sum_{j=1}^{3} W_{j} q s_{j}+\sum_{k=1}^{1} W_{k} s c_{k}$

The equation (1) includes the context information of the user's mobile phone. The equation (2) shows the QoS information and the equation (3) determines the context information of service provider. In addition, in the equation (4), $w_{i}, w_{j}$ and $w_{k}$ are the assigned weights to $c c_{i}, q s_{j}$ and $\mathrm{sc}_{\mathrm{k}}$ respectively which show the degree of their importance in service selection. $w_{i}$ is extracted from the user profile on the mobile phone. In case of a VIP user, $w_{k}$ will be greater than zero. This indicates that the service selection process can be executed more carefully.

Consequently, seven parameters are considered for determining the solution optimality.

According to the equation (5), a formula based on the total weight of all parameters is used to calculate the fitness value of a solution and best service selection.

\subsection{The Result of Evaluation}

Regarding to the limited similar works in this area, VOLARE system is intended as a base of comparison. Thus, CSRAM and VOLARE frameworks were executed on the defined data set and their outputs were compared. The execution parameters are as below:

$$
\begin{aligned}
& W_{i} \in[0.05,0.05,0.05], \mathrm{i} \in[1 . .3] \\
& W_{j} \in[0.2,0.2,0.2], \mathrm{j} \in[1 . .3] \\
& W_{k} \in[0.25], \mathrm{k}=1
\end{aligned}
$$

Number of executions at different time instances: 20

The Iteration number of genetic algorithm: until obtaining a solution

The iteration number of adaptation process: 24

The number of population members at any given moment: 256

The rate of Crossover: 0.005

The rate of Mutation: 0.005

The selection size: 0.75

It should be mentioned that the average value of the optimum solutions obtained from every 20 iterations at any given moment is considered as the final solution.

Figure 5 shows the inputs, and also the outputs of the amount of money saving in service cost for both approaches. In this graph, horizontal axis determines the test duration based on minutes and seconds and the vertical axis shows the percent value of each input parameter and related results. As you see, VOLARE framework assumes that the subscriber has chosen $256 \mathrm{kbps}$ service mode. Now, regarding to the four service modes $(28,56,128$ or $256 \mathrm{Kbps})$ and based on the battery, the 
available network and response bandwidth, the user should pay $\% 25, \% 50, \% 75$ or $\% 100$ of the cost. Therefore, the comparison graph has been designed based on the above three parameters, but in fact, considering seven different parameters in CSRAM framework has increased adaptability and flexibility of this approach in reality; as in most test epochs, approximately the same cost efficiency as VOLARE approach was achieved.

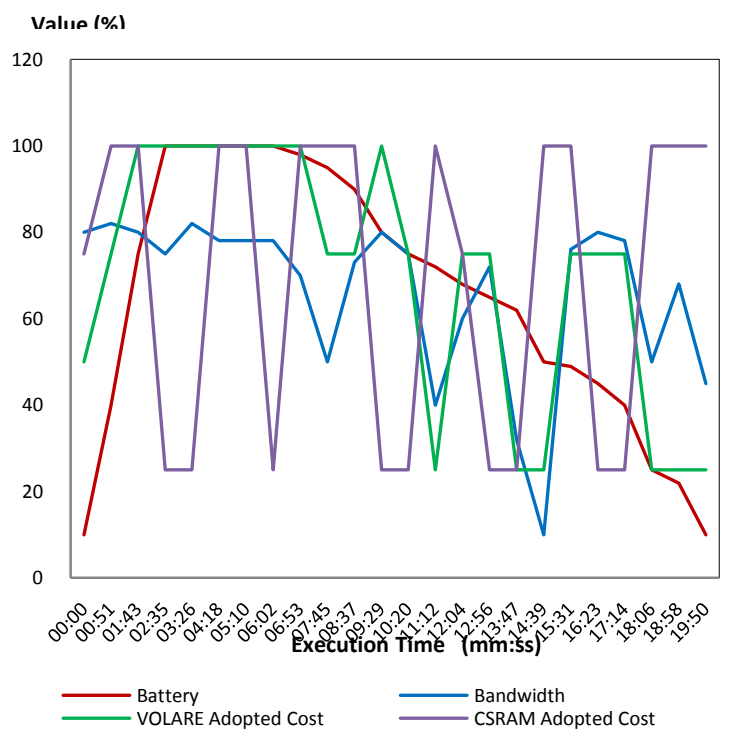

Fig 5: Percentage of saving in cost in compare with VOLARE

Table 1 illustrates the obtained evaluation results regarding to different parameters. In this table, the parameter values of the selected solution are shown.

As told before, some advantages of CSRAM framework are its Efficiency for solving the problems in the different practical scopes as well as its flexibility in variable QoS parameters. The result of comparing between the proposed frameworks with VOLARE approach indicates the ability of CSRAM approach in obtaining more optimal solutions. Table 2 shows the number of considered parameters and the related results about each approach.

\section{CONCLUSION AND FURTHER WORKS}

Today, using mobile technology in cloud computing is at the center of some different researcher's attention, because of the complexity of the environment and its usability feature. In mobile cloud computing systems, there are a lot of users and also a variety of services. The heterogeneity of service and the user's different conditions leads to the consideration of several parameters in the process of the optimum service selection. Therefore, in this article, it was tried to consider all the important criteria by proposing an effective framework that covers the above mentioned homogeny. In addition, CSRAM, in comparison with similar previous frameworks, has decreased the amount of imposed calculations in the user's mobile phone and it can consider the different environmental parameters for obtaining results with higher precision; also, flexibility of the proposed framework make it possible to use other optimization algorithm rather than genetic algorithm and to change the evaluation criteria.

Results of comparison between CSRAM framework and another framework on the intended data set showed that the proposed framework has achieved results approximately similar to those of the above mentioned framework by reducing the cost of usage $\% 30$ less than what agreed in the SLA with regard to more environmental parameters. This proves that the generated solution of CSRAM framework is more accurate.

Finally, the next phase of this research will be the open issue of the roll of failure recovery process on different modules of CSRAM framework and also, the effect of using other environmental parameters on the evaluation results.

Table 1. the values of involved parameters in CSRAM

\begin{tabular}{|c|c|c|c|c|c|c|c|}
\hline $\begin{array}{c}\text { Time } \\
\text { (mm:ss) }\end{array}$ & 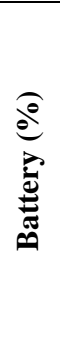 & 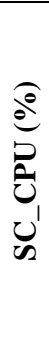 & 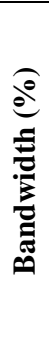 & 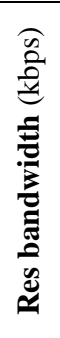 & 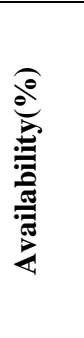 & 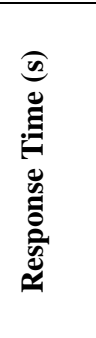 & 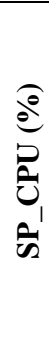 \\
\hline 00:00 & 10 & 45 & 80 & 122 & 99.29 & 0.4318 & 45 \\
\hline 00:51 & 40 & 68 & 82 & 247 & 99.66 & 0.0445 & 68 \\
\hline 01:43 & 75 & 50 & 80 & 209 & 95.88 & 0.0866 & 50 \\
\hline $02: 35$ & 100 & 78 & 75 & 21 & 99.87 & 0.0175 & 78 \\
\hline 03:26 & 100 & 80 & 82 & 18 & 99.76 & 0.1938 & 80 \\
\hline 04:18 & 100 & 10 & 78 & 246 & 99.84 & 0.1104 & 10 \\
\hline 05:10 & 100 & 32 & 78 & 253 & 99.98 & 0.0055 & 32 \\
\hline 06:02 & 100 & 72 & 78 & 22 & 99.97 & 0.0339 & 72 \\
\hline $06: 53$ & 98 & 60 & 70 & 249 & 99.88 & 0.0922 & 60 \\
\hline 07:45 & 95 & 40 & 50 & 189 & 99.99 & 0.1026 & 40 \\
\hline 08:37 & 90 & 75 & 73 & 208 & 99.99 & 0.0894 & 75 \\
\hline 09:29 & 80 & 80 & 80 & 15 & 99.34 & 0.1687 & 80 \\
\hline $10: 20$ & 75 & 73 & 75 & 21 & 99.95 & 0.2095 & 73 \\
\hline $11: 12$ & 72 & 50 & 40 & 254 & 99.50 & 0.0887 & 50 \\
\hline 12:04 & 68 & 70 & 60 & 102 & 99.99 & 0.0006 & 70 \\
\hline $12: 56$ & 65 & 78 & 72 & 27 & 99.93 & 0.0773 & 78 \\
\hline $13: 47$ & 62 & 78 & 32 & 18 & 99.47 & 0.0172 & 78 \\
\hline $14: 39$ & 50 & 78 & 10 & 243 & 99.97 & 0.0076 & 78 \\
\hline $15: 31$ & 49 & 82 & 76 & 233 & 99.96 & 0.2310 & 82 \\
\hline $16: 23$ & 45 & 75 & 80 & 23 & 99.67 & 0.0051 & 75 \\
\hline $17: 14$ & 40 & 80 & 78 & 26 & 99.83 & 0.0158 & 80 \\
\hline 18:06 & 25 & 82 & 50 & 234 & 100 & 0.3547 & 82 \\
\hline 18:58 & 22 & 80 & 68 & 234 & 99.99 & 0.2666 & 80 \\
\hline $19: 50$ & 10 & 68 & 45 & 222 & 99.97 & 0.0712 & 68 \\
\hline
\end{tabular}


Table 2. VOLARE framework vs CSRAM framework

\begin{tabular}{|c|c|c|c|}
\hline & $\begin{array}{c}\text { Involved } \\
\text { Parameters }\end{array}$ & $\begin{array}{c}\text { Percentage } \\
\text { of Saving }\end{array}$ & $\begin{array}{c}\text { Adaptation } \\
\text { Model }\end{array}$ \\
\hline $\begin{array}{c}\text { VOLARE } \\
\text { Framework }\end{array}$ & 3 & $30 \%$ & $\begin{array}{c}\text { Mathematical } \\
\text { Alg. }\end{array}$ \\
\hline $\begin{array}{c}\text { CSRAM } \\
\text { Framework }\end{array}$ & 7 & $30 \%$ & Genetic Alg. \\
\hline
\end{tabular}

\section{REFERENCES}

[1] Fernando, Niroshinie, W.Loke, Seng, Rahayu, Wenny, 2013 Mobile cloud computing: A survey, Future Generation Computer Systems.

[2] Papakos, Panagiotis, Capra, Licia, Rosenblum, David S, 2010. VOLARE: Context-Aware Adaptive Cloud Service Discovery for Mobile Systems, ARM.

[3] Renders, J.M., Flasse, S.P., 1996. Hybrid methods using genetic algorithms for global optimization. IEEE Trans. Syst. Man Cybern. Part B, 26(2):243-258.

[4] Ghorbannia Delavar, Arash, Aryan, Yalda, 2011. A Synthetic Heuristic Algorithm for Independent Task Scheduling in Cloud Systems. IJCSI International Journal of Computer Science Issues, Vol. 8, Issue 6, No 2, November (2011) ISSN (Online): 1694-0814.

[5] Ghorbannia Delavar, Arash, Aryan, Yalda, 2012. A Goal-Oriented Workflow Scheduling in Heterogeneous Distributed Systems. International Journal of Computer Applications (0975 - 8887) Volume 52 - No. 8.

[6] T. Dinh, Hoang, Lee, Chonho, Niyato, Dusit, Wang, Ping. 2011. A survey of mobile cloud computing: Architecture, Applications, and Approaches, Wireless Communications and Mobile Computing.

[7] Capra L., Emmerich W., Mascolo C. . 2003. CARISMA: contextaware reflective middleware system for mobile applications.Software Engineering, IEEE Transactions on, 29(10):929-945.

[8] Keeney J., Cahill V., 2003. CHISEL: A Policy-Driven, Context-Aware, Dynamic Adaptation Framework, Fourth IEEE International Workshop on Policies for Distributed Systems and Networks (POLICY 2003), 4-6 June 2003; $3-14$

[9] Rouvoy R, Barone P, Ding Y, Eliassen F, Hallsteinsen S,Lorenzo J, Mamelli A, Scholz U., 2009. MUSIC: middleware support for self-adaptation in ubiquitous and service-oriented environments, Software Engineering Self Adaptive Software Systems LNCS 5525:164-182.

[10] Bertolino A., Emmerich W., Inverardi P., Issarny V., Liotopoulos F., Plaza P., 2009. PLASTIC: Providing Lightweight Adaptable Service Technology for Pervasive Information \& Communication, Automated Software Engineering - 23rd IEEE/ACM International Conference on Bertolino.

[11] Capra L., Zachariadis S. and Mascolo C., 2005. Q-CAD: QoS and Context Aware Discovery Framework for Adaptive Mobile Systems, In Proc. of IEEE Int Conference on Pervasive Services (ICPS05), Santorini, Greece.

[12] Mukhija A., Dingwall-Smith A., Rosenblum D. S., 2007. QoSAware Service Composition in Dino (ECOWS.2007), 5th European Conference on Web Services.

[13] Song, Hyewon, Bae, Chang Seok, Lee, Jeun Woo, Youn, Chan-Hyun, 2011. Utility Adaptive Service Brokering Mechanism for Personal Cloud Service, Military Communications Conference.

[14] Soukkarieh, Bouchra, Sèdes, Florence, 2009. Dynamic Services Adaptation to the User's Context, Fourth International Conference on Internet and Web Applications and Services.

[15] Li, Fei, Rasch, Katharina, Truong, Hong-Linh, Ayani, Rassul, Dustdar, Schahram, 2010. Proactive Service Discovery in Pervasive Environments, ICPS.

[16] Garg, Saurabh Kumar, Versteeg, Steve, Buyya, Rajkumar, 2012. A framework for ranking of cloud computing services, Future Generation Computer Systems Journal.

[17] La, Hyun Jung, Kim, Soo Dong, 2010. A Conceptual Framework for Provisioning Context-aware Mobile Cloud Services, IEEE 3rd International Conference on Cloud Computing.

[18] Zhang, Peng, Yan, Zheng, 2011. A QoS-AWARE SYSTEM FOR MOBILE CLOUD COMPUTING, Proceedings of IEEE CCIS.

[19] Qing, Wu, Zhenbang, Li, Yuyu, Yin, Hong, Zeng, 2012. Adaptive Service Selection Method in Mobile Cloud Computing, China Communications. 